\title{
Metamaterial based Flexible Coplanar Antenna Design and Simulation for Human Body Applications
}

\author{
Gamze TETİK ${ }^{1}$, Erkan TETIK ${ }^{2 *}$
}

\begin{abstract}
This study presents a metamaterial based flexible coplanar antenna designed to operate close to the human model in the $2.45 \mathrm{GHz}$ operation frequency band. Firstly, the reflection values and radiation pattern of the suggested antenna were analyzed. After obtaining good results, electromagnetic band gap (EBG) structure that is a kind of metamaterials was designed. Secondly, the EBG and the antenna was combined to form an integrated structure. At the same time, a human model was designed for the integrated structure. Conductive textile fabrics such as pure copper polyester taffeta fabric and felt were used for coplanar antenna and EBG design, respectively. Finally, the specific absorption rate (SAR) values of the coplanar wearable antenna and the integrated model were separately computed. As a result, the proposed EBG structure effectively reduced the SAR value of the integrated model. It was seen that the SAR value of integrated model was suitable with the standard threshold. The originality of the work lies in the use of wearable textile materials and making calculation by applying bend to the proposed structure. In addition, the sharp drop in SAR value from 31.8 to $0.0344 \mathrm{~W} / \mathrm{kg}$ is remarkable when compared to many studies in the literature. The proposed integrated design has potentials to be applied to many research areas such as the military systems, health applications, and e-textile technologies.
\end{abstract}

Keywords: Flexible antenna, metamaterial, human body, SAR

${ }^{1}$ Gamze TETIKK (Orcid ID: 0000-0002-5968-7244), Usak University, Faculty of Engineering, Materials Science and Nanotechnology Engineering, Usak, Turkey

${ }^{2}$ Erkan TETIK (Orcid ID: 0000-0002-8183-8141), Usak University, Faculty of Education, Computer Education and Instructional Technology, Usak, Turkey

*Corresponding Author: Erkan TETİK, e-mail: erkan.tetik@usak.edu.tr

Geliş tarihi / Received: 13-02-2020

Kabul tarihi / Accepted: 18-08-2020 


\section{INTRODUCTION}

The refraction index is defined as the measure of bending of a light ray during the transfer from one medium into another. There are limited studies considering the refraction index as negative and these studies are listed in detail by Moroz (Moroz, 2009). Veselago first theorized that a medium can have negative refractive index that is expressed by the permittivity and permeability parameters (Veselago, 2002). These parameters determine the electromagnetic (EM) properties. It was experimentally proved that split ring resonators can have effective negative permittivity and permeability parameters simultaneously at particular frequency ranges between the years of 1996-1999 (Pendry et al., 1999), and these artificial materials were expressed as metamaterials (MTMs). MTMs that are defined as artificial EM materials have unnatural features like negative refraction and formations of them enabled free control of the EM parameters. These structures were experimentally investigated as single compact structures in one (Pendry, 2000) and two (Shelby et al., 2001) dimensions in 2000 and 2001, respectively. These studies guided the researchers to focus on MTMs and thus the researches on them increased remarkably (Ozbay and Aydin, 2008 Almoneef and Ramahi, 2014; O. Akgol et al., 2017). MTMs have many application areas such as cloaking (Alu and Engheta, 2008), absorber (Bagmanci et al., 2019; Bakır et al., 2016; Bağmancı et al., 2017), harvesting (Xiao et al., 2020; Oguzhan Akgol et al., 2017; Bakır, 2018), sensor (Bakır et al., 2015; Tetik and Erdiven, 2018), and chiral (Sabah et al., 2015). In addition, they can be used in the integrated antennas together with the electromagnetic band gap (EBG) structures that are kinds of MTMs (Abdulhameed et al., 2020; Bai and Langley, 2009; Tetik and D. Tetik, 2018). EBG designs have a characteristic to suppress EM wave propagation in operation frequency ranges and hence these structures provide a reduction in the radiation which is produced by the antennas. Since it reduces the radiation absorbed by the human body, researches on EBG integrated with the antennas ascended.

With the developments in wireless communication technologies that can be integrated into human body, the electro-textile (e-textile) materials attracted considerable attention (Salonen et al., 2004; A. Tronquo et al., 2006; Anneleen Tronquo et al., 2006; Soh et al., 2012). These materials can be integrated into electronic devices that can be applied in fields such as the body worn communication technologies, health monitoring, military, and textile sciences. One of the most remarkable of these fields is wearable wireless communication technology field and it can be defined as the link between wirelessly communicating devices that are body-mounted. An important part of these integrated devices consists of fabric-based wearable antenna (WAs) types. WAs which are described as the e-textile based antenna devices have many advantages such as inexpensiveness, washability, lightness, and ability to be hidden. As these structures can be easily associated into everyday clothes, it attracts researchers' attention and there are many investigations about the wearable e-textile antennas. In addition, the studies demonstrated that the WAs are flexible and this means low and stable resistivity that should be less than $1 \Omega / \mathrm{sq}$. The e-textile based antennas usually consist of a substrate that is produced with materials such as felt, cotton, flannel fabric, and jeans. In addition to this material, the pure copper polyester taffeta fabric (PCPTF) is usually used as a conductor (Zhu and Langley, 2009; Gupta et al., 2010). Researchers focused on designing EBG structures with these materials due to the advantages of e-textile based applications (Sankaralingam and Gupta, 2010). They also improved integrated structures consisting of antenna and EBG. By the new technologies, the number of wearable wireless devices is rapidly increasing. One of the most important disadvantages of the WAs is their closeness to human body. The human body may be exposed to antenna radiation due to contact and it may be negatively affected owing to the amount of power absorbed per unit mass of the body that is expressed by the specific absorption rate (SAR). In this 
context, these integrated structures have the feature of reducing the backward radiation reflecting from the antenna. The current paper aims to minimize the EM radiation caused by wireless devices on the human body.

Firstly, we designed the coplanar textile antenna that can operate at $2.45 \mathrm{GHz}$ and investigated its reflection coefficients and radiation pattern. Then, the suggested antenna and MTM based EBG structure were designed to be integrated. After obtaining the desired values, the proposed design was created by integrating the antenna and EBG structure. Afterwards, we designed the cylindrical human tissue model for the SAR value analysis. We obtained the SAR value of the suggested WA and the integrated model using the proposed human model. Finally, we investigated the effect on human body of the suggested WA and integrated structures.

\section{MATERIALS AND METHODS}

We designed the proposed antenna and EBG model using the e-textile materials that were indispensable for WA applications. These materials are produced from conductive fabrics that are obtained by interleaving traditional fabrics with conductive metal/polymer threads. The PCPTF has $0.031 \Omega / \mathrm{sq}$ measured resistance, $2.5 \times 10^{5} \mathrm{~S} / \mathrm{m}$ electrical conductivity, and $0.05 \Omega / \mathrm{sq}$ surface resistance values. Therefore, it was preferred as conductive material in order to maintain sufficient conductivity and flexibility. For substrate part of models, we used the felt textile material that has 0.016 loss tangent, 1.22 permittivity, and $0.65 \mathrm{~mm}$ thickness. In order to perform the electrical characterization (permittivity $\varepsilon$ and loss tangent $\tan \delta$ ) of felt fabric, two different methods are used (Mantash et al., 2012): the method of stub resonator (Liu et al., 2009) which is valid for a single frequency and a broadband method based on a stripline cell (Salahun et al., 2001). The geometry of the suggested wearable coplanar antenna designed using these e-textile materials is demonstrated in Fig. 1. The designed antenna that had sizes of $\mathrm{w}=41$ and $\mathrm{h}=46 \mathrm{~mm}$ consists of 3 parts as the patch, substrate, and ground plane. The coplanar patch and ground plane parts of antenna were designed using the PCPTF material that had a thickness of $0.035 \mathrm{~mm}$.

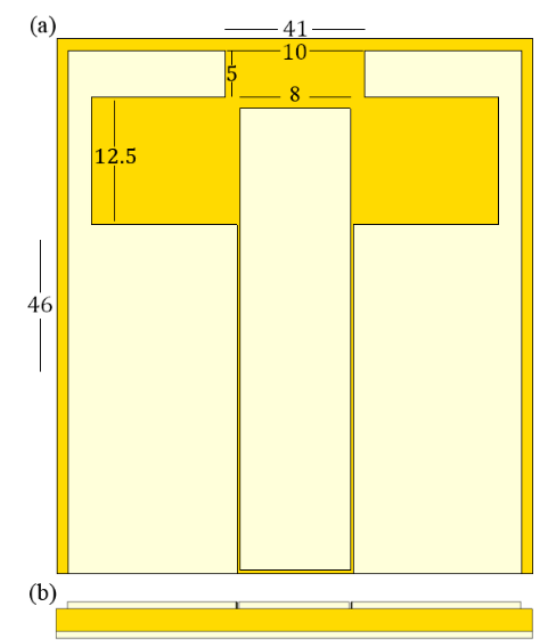

Figure.1. The suggested coplanar flexible antenna (a) top view (b) side view.

To design a MTM based low-profile integrated structure, we designed the EBG structure that was used to achieve phase reflection in the $2.45 \mathrm{GHz}$ operation frequency. The proposed EBG structure which consists of more than one-unit cell was designed as a ground plane for the coplanar WA. The unit cell of this EBG structure is demonstrated Fig. 2. This unit cell consisted of a combination of conductors and dielectrics arranged in either a single row or multiple columns. The EBG structures consisting of such unit cells can exhibit very high surface impedance at particular frequency ranges. In this context, 
we used the proposed EBG structure to block EM waves in resonance frequency value of the proposed coplanar flexible antenna. We preferred the PCPTF and felt material in a similar way to the proposed EBG structure. The EBG and coplanar antenna design using e-textile materials had as good results as conventional antenna designs. The suggested EBG structure that had dimensions of $30 \times 30 \mathrm{~mm}$ consists of 3 parts as the patch, substrate, and ground plane. The substrate that had $2 \mathrm{~mm}$ thickness was made of felt material. The patch and ground plane were made of PCPTF material.

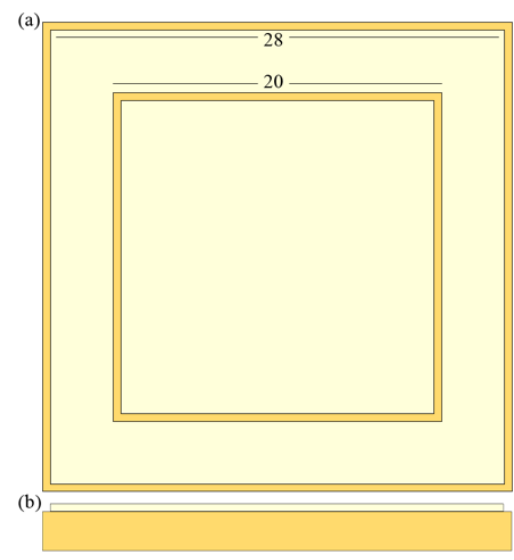

Figure.2. The MTM based EBG structure (a) top view (b) side view.

We investigated the SAR characteristics of the proposed e-textile based structures by using a fullwave EM solver CST Microwave Studio (Computer Simulation Technology GmbH, Darmstadt, Germany) based on the finite integration technique (FIT). CST Microwave Studio is a high-performance 3D EM analysis software package for designing, analyzing and optimizing EM components and systems. Common subjects of EM analysis include the performance and efficiency of antennas, electromagnetic compatibility, exposure of the human body to EM fields, energy harvesting, and sensor devices.

\section{RESULTS AND DISCUSSION}

We realized the calculation and designs of the proposed model which consisted of the WA, the EBG structure, and the antenna-EBG based integrated structure. After designing structures, we simulated to obtain the ideal dimensions of proposed antenna and performed to analyze the S-Parameters (Magnitude in $\mathrm{dB}$ ) and surface current distribution. The periodic structures such as EBG used in the microwave applications can exhibit the pass band or stop band characteristics. We obtained the absorption and permittivity properties of the EBG structure to analyze its usage with the proposed antenna. The calculation studies of suggested models were performed at 1-3.4 GHz frequency range.
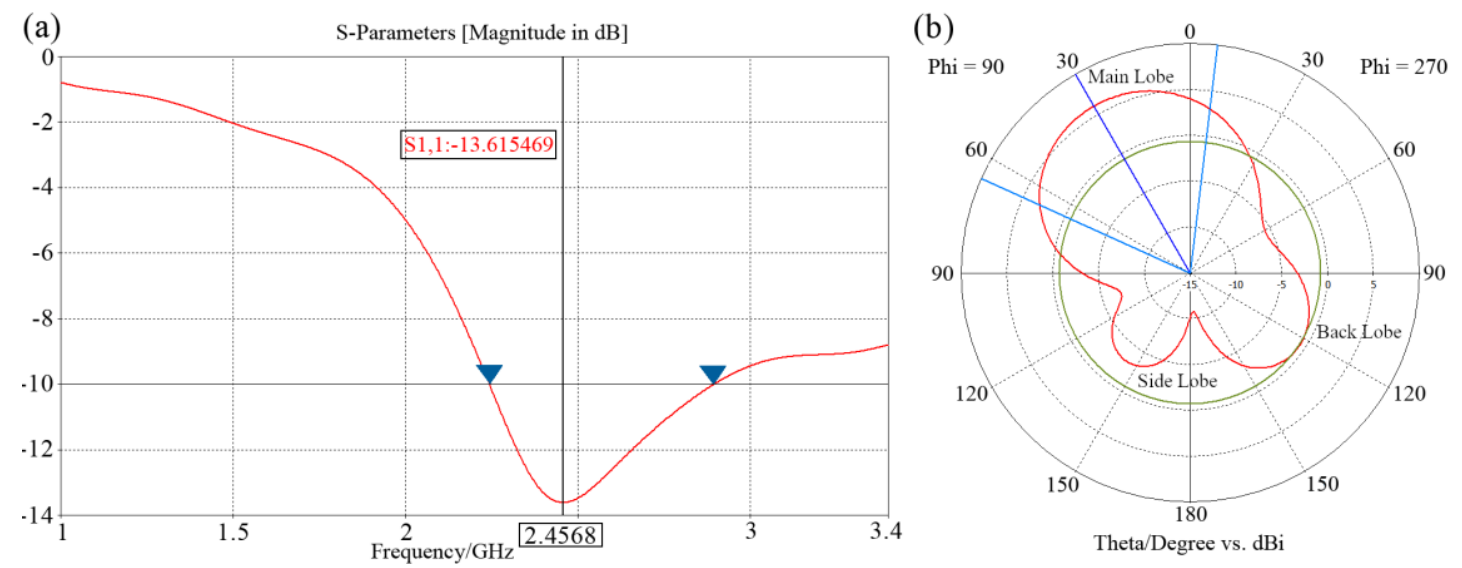

Figure.3. The reflection coefficient (a) and radiation pattern (b) of the proposed coplanar antenna. 
We investigated the performance of proposed wearable coplanar antenna in the $2.45 \mathrm{GHz}$ industrial, scientific, and medical (ISM) band and obtained the reflection coefficient from calculations that is demonstrated in Fig. 3(a). The proposed antenna reflected at $2.454 \mathrm{GHz}$ frequency value and had a remarkable propagation. We obtained the peak value of $-13.4568 \mathrm{~dB}$ for the $\mathrm{S}_{11}$. The frequency range in which an antenna operates can be measured by means of the reflection coefficient $\left(\left|S_{11}\right|^{2}\right)$. This coefficient provides information about the behavior of the antenna. For a standard antenna, the incident wave (a) will be reflected (b) and radiated (c). In this case, the reflection coefficient is defined as $\left|S_{11}\right|^{2}=$ $|b|^{2} /|c|^{2}$ and is expressed in $d B$, thus as $A=10 \log \left|S_{11}\right|^{2}$. The outcome provides information on the performance of the antenna because $\mathrm{A}<-10 \mathrm{~dB}$ means that no more than $10 \%$ of the incoming power is reflected by the antenna, whereas at least $90 \%$ is radiated when no losses occur. An antenna having these properties can be considered of good quality. In this context, we obtained the bandwidth of the proposed antenna between the point where $-10 \mathrm{~dB}$ line cuts the curve in Fig. 3(a). The first and second points which give the low and high frequencies have 2.24 and $2.91 \mathrm{GHz}$ values, respectively. The bandwidth of the proposed antenna has $0.67(2.91-2.24) \mathrm{GHz}(670 \mathrm{MHz})$ value. In this case, it was understood that this coplanar antenna can be efficiently applied in wearable technologies where e-textile applications have a dominant importance. Then, we computed the radiation pattern of the suggested coplanar antenna that is shown Fig. 3(b) at the operation frequency band range. This radiation pattern has the directional characteristics that is characterized by more efficient radiation in one direction than another. In addition, it has three lobes called main, side and back. The main lobe has maximum gain of $5.97 \mathrm{dBi}$ at proposed frequency. According to the results obtained, e-textile based proposed coplanar antenna can be easily used in industrial applications via its geometry and frequency band value.

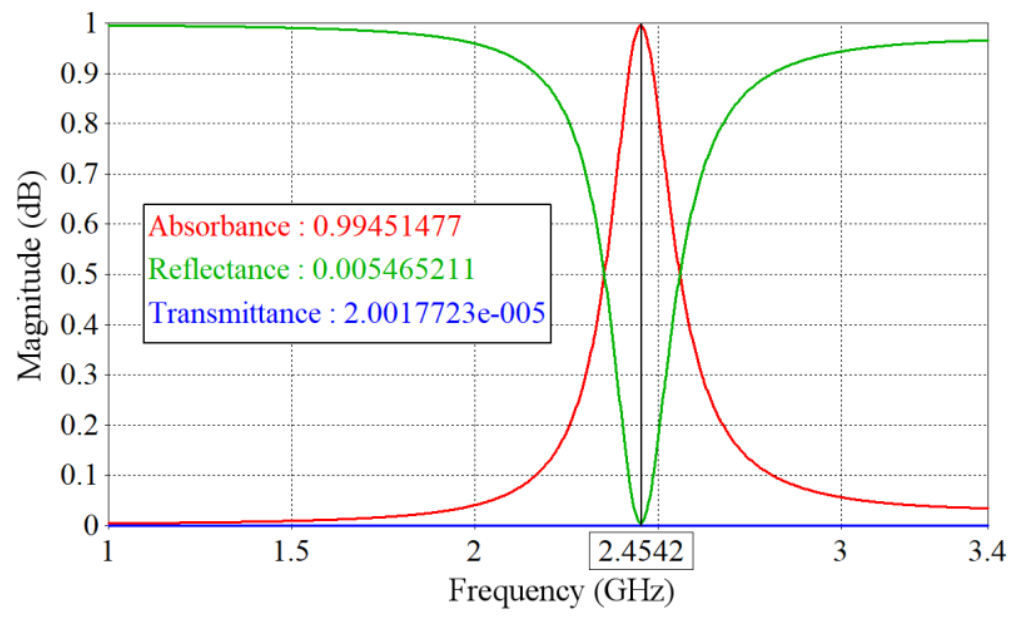

Figure.4. The absorbance, reflectance, and transmittance results of the proposed EBG structute.

After the design and simulation procedure the proposed coplanar antenna, we achieved the absorption, reflection, and transmission characteristics of EBG structure. These simulations are carried out at 1-3.4 GHz frequency range. The calculation results obtained are shown in Fig. 4. The absorption characteristic of these structures is related to its transmission $\mathrm{T}(\omega)=\left|S_{21}\right|^{2}$ and reflection $\mathrm{R}(\omega)=$ $\left|S_{11}\right|^{2}$, according to the formula: $\mathrm{A}(\omega)=1-\mathrm{R}(\omega)-\mathrm{T}(\omega)$. According to this formula, maximizing frequency value of the absorption $\mathrm{A}(\omega)$ is equivalent to minimizing simultaneously both the transmission $(T(\omega))$ and the reflectivity $(R(\omega))$ at the same frequency range. In order to maximize the absorption, the reflection and transmission, which are expressed by the equation $R(\omega)=\left|S_{11}\right|^{2}$ and $\mathrm{T}(\omega)=\left|\mathrm{S}_{21}\right|^{2}$, should be minimized at the resonance frequency. In order to completely prevent transmission of the structure, its back site is blocked with a PCPTF e-textile material. In this context, the 
calculation results of the EBG structure have been investigated according to the absorption equation $\mathrm{A}(\omega)=1-\mathrm{R}(\omega)$ where $\left|\mathrm{S}_{21}\right|^{2}=0$ since $\mathrm{T}(\omega) \rightarrow 0$. According to the results obtained, the transmission value is zero at the all frequency values and reflection only has a minimum value at the resonance frequency that is $2.45 \mathrm{GHz}$. The absorption which is of about $99.45 \%$ reaches its maximum value at the resonance frequency. As a results, the proposed EBG structure can be used to suppress EM wave propagation at $2.45 \mathrm{GHz}$ ISM. In addition, this EBG structure can be applied to all antennas working at the proposed ISM frequency.
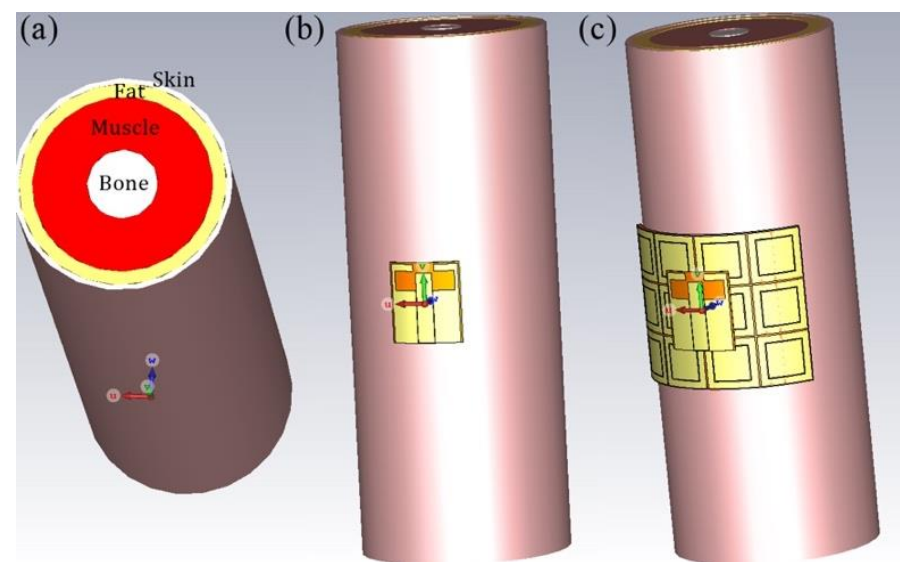

Figure.5. The human model (a), the proposed wearable coplanar antenna in direct contact with the human model (b), and the proposed integrated structure and human model.

Once we achieved the ideal antenna and EBG structures, we created the cylindrical human model that is demonstrated in Fig. 5(a). The suggested human design consisted of the bone, muscle, fat, and skin tissue layers that had 13,20,5, and $2 \mathrm{~mm}$ radii, respectively. These tissues were located in a cylinder human model that had $40 \mathrm{~mm}$ total radius. The proposed antenna was integrated into this human model so that calculations were performed. It is demonstrated in Fig. 5(b) that the analyzed WA was in direct contact with this human body. Then, proposed integrated antenna and EBG structure was placed on human model that had same design. The antenna and human model design in the Fig. 5(b) led significant radiation problems. To overcome radiation problems, we placed the proposed EBG structure between the coplanar antenna and human model as depicted in Fig. 5(c) and realized the bending to both models. The EBG structure integration was performed to control the SAR value.
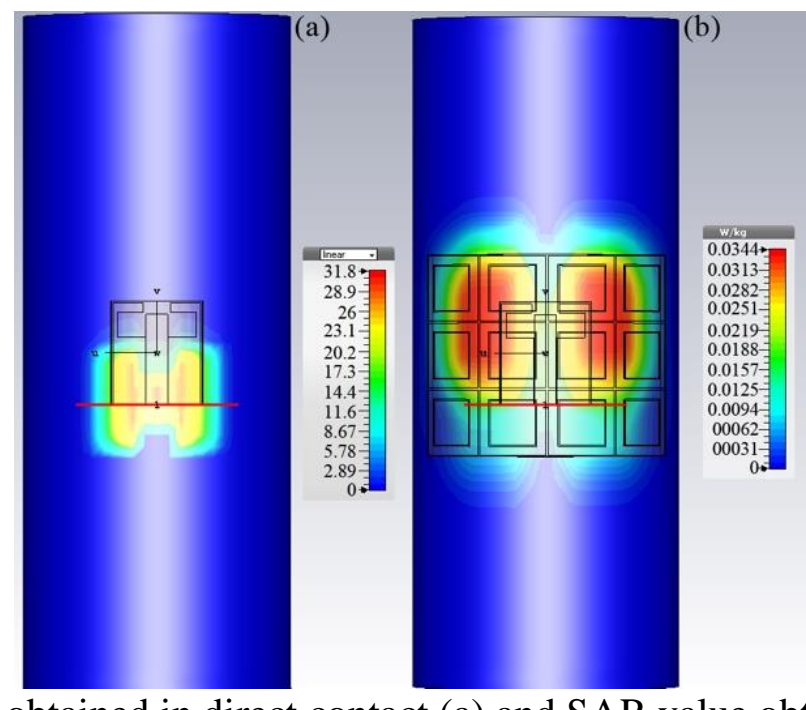

Figure.6. The SAR value obtained in direct contact (a) and SAR value obtained when EBG structure was placed between human body and antenna (b). 
We performed the SAR calculations for the suggested MTM based wearable coplanar antenna. The SAR calculations were used for controlling the effects of potential health hazard on the human body. The SAR value is expressed as follows

$\mathrm{SAR}=\mathrm{P} / \sigma=\sigma \mathrm{E}^{2} / 2 \rho$

where $\sigma$ is the tissue conductivity, $\mathrm{P}$ is the power loss density, $\mathrm{E}$ is the electric filed amplitude in tissue, and $\rho$ is the density of tissue. After SAR analysis, the SAR reduction factor (SRF) value was calculated for proposed structures, that was defined by equation 2 .

$\operatorname{SRF}(\%)=\left(\mathrm{SAR}_{\text {antenna }}-\mathrm{SAR}_{\text {antena }+\mathrm{EBG}} / \mathrm{SAR}_{\text {antenna }}\right) \times 100$

In this context, the amount of EM wave absorbed in the human body due to suggested WA was investigated. According to the analysis results of the proposed structures, we obtained the SAR value as $31.8 \mathrm{~W} / \mathrm{kg}$ when there was a direct contact between the human model and coplanar antenna as demonstrated in Fig 6(a). This SAR value might cause the health risks on the human body. The SAR results of proposed EBG structure based integrated structure are demonstrated in Fig. 6(b). The proposed EBG design had $4 \times 3$ unit cell. In this case, the SAR value of this structure decreased to $0.0344 \mathrm{~W} / \mathrm{kg}$. The proposed structure was remarkably impressive considering that this result was so lower than the European standard ( $2 \mathrm{~W} / \mathrm{kg}$ ). In addition, the SRF value was calculated as $99.89 \%$ using the obtained SAR value for integrated structure. It was found that the suggested model had a quite good surface wave suppression characteristic.

Table 1. Frequency, antenna locations, and SAR values in the present study and the other studies.

\begin{tabular}{lccc}
\hline Studies (10 g tissue) & $\begin{array}{c}\text { Frequency } \\
(\mathbf{G H z})\end{array}$ & Location & SAR (W/Kg) \\
\hline Present Study & 2.45 & Human arms & 0.0344 \\
Afridi et al. & 2.4 and 5.2 & Human arms & 1.90 and 0.92 \\
Yan et al. & 2.4 & Human arms (mode 1 and 2) & 0.05 and 0.01 \\
Kim et al. & 6 & Human wrist & 0.15 \\
\hline
\end{tabular}

Table 1 shows the results of the calculation, together with the present study and the other studies. Afridi et al. presented two type of dual band $(2.4$ and $5.2 \mathrm{GHz})$ wearable planar dipole antenna. They obtained 1.90 and $0.92 \mathrm{~W} / \mathrm{kg}$ simulated SAR values for 2.4 and $5.2 \mathrm{GHz}$ frequency bands, respectively. These values are at a high level compared to the literature. Yan et al. realized a pattern-reconfigurable WA design based on MTM structure. They achieved the simulated SAR value of 0.05 and $0.01 \mathrm{~W} / \mathrm{kg}$ for mode 1 and 2. These values are quite remarkable when the European standard threshold was considered. Kim et al. designed a reconfigurable bean-steering patch antenna for wrist-wearable applications. They achieved the SAR values in the range of 0.08 and $0.15 \mathrm{~W} / \mathrm{kg}$. These results are also remarkable, but they are outside the ISM band range. In this study, the SAR value in the $2.45 \mathrm{GHz}$ frequency band was obtained as $0.0344 \mathrm{~W} / \mathrm{kg}$. These results are quite significant compared to many values in the literature.

Wearable electronics devices are leading thanks to advancements in miniature electronics fabrication, wireless communications, and batteries. Not only for personal life such as mobile phones and smartwatches, but also for providing a wider range of services such as indoor positioning, physical and mental health monitoring, and sports analytics. On the other hand, these devices harm human health due to EM radiation they caused. The proposed structure can be used by integrating with devices such as the mobile phones, tablets, and smartwatches, so that the damage will be minimized. Consequently, 
the proposed MTM based WA can be utilized in various applications where the e-textile antenna technologies are used.

\section{CONCLUSIONS}

In this study, the EBG based wearable circular antenna that operated at $2.45 \mathrm{GHz}$ frequency band was investigated. The e-textile based antenna was designed and its reflection coefficients were simulated. The results obtained from the simulations demonstrated that this antenna reflected at 2.45 $\mathrm{GHz}$ frequency. We performed the SAR analysis of the proposed structure according to these results. We designed the MTM based EBG structure and integrated it to this antenna. Similarly, we obtained the SAR results of this integrated structure. The proposed coplanar antenna directly mounted on the body gave $31.8 \mathrm{~W} \mathrm{kg-1} \mathrm{SAR} \mathrm{value} \mathrm{taken} \mathrm{over} 10 \mathrm{~g}$ of tissue. This value was very high for European standard ( $2 \mathrm{~W} \mathrm{~kg}-1$ ). To overcome the potential health hazard, the proposed integrated model was designed using antenna and EBG structures. Thus, this integrated structure reduced the value of SAR to $0.0344 \mathrm{~W} \mathrm{kg-}$ 1. This value was remarkable according to the acceptable SAR value standards. Moreover, the proposed integrated model had $99.89 \% \mathrm{SRF}$ values for 4x3 EBG structure. This value was quite adequate for the WA applications. Consequently, the proposed integrated antenna design can be applied to many research areas such as mobile devices, military systems, health applications, and e-textile technologies.

\section{ACKNOWLEDGEMENTS}

This study was supported by Usak University Scientific Research Foundation (Project Number: 2018/SOSB003).

\section{REFERENCES}

Abdulhameed MK, Isa MSB, Zakaria Z, Ibrahim IM, Mohsen MK, Attiah ML, Dinar AM, 2020. Enhanced performance of compact $2 \times 2$ antenna array with electromagnetic band-gap. Microwave and Optical Technology Letters, 62(2): 875-886.

Afridi A, Ullah S, Khan S, Ahmed A, Khalil AH, Tarar MA, 2013. Design of Dual Band Wearable Antenna Using Metamaterials. Journal of Microwave Power and Electromagnetic Energy, 47(2), 126-137.

Akgol O, Altintas O, Dalkilinc EE, Unal E, Karaaslan M, Sabah C, 2017. Metamaterial absorber-based multisensor applications using a meander-line resonator. Optical Engineering, 56(8): 087104.

Akgol O, Bağmancı M, Karaaslan M, Ünal E, 2017. Broad band MA-based on three-type resonator having resistor for microwave energy harvesting. Journal of Microwave Power and Electromagnetic Energy, 51(2): 134-149.

Almoneef T, Ramahi OM, 2014. A 3-Dimensional Stacked Metamaterial Arrays For Electromagnetic Energy Harvesting. Progress In Electromagnetics Research, 146: 109-115.

Alu A, Engheta N, 2008. Plasmonic and metamaterial cloaking: Physical mechanisms and potentials. Journal of Optics A: Pure and Applied Optics, 10(9): 093002

Bağmancı M, Karaaslan M, Ünal E, Akgol O, Bakır M, Sabah C, 2019. Solar energy harvesting with ultra-broadband metamaterial absorber. International Journal of Modern Physics B, 33(08): 1950056.

Bağmanc1 M, Karaaslan M, Ünal E, Akgol O, Karadağ F, Sabah C, 2017. Broad-band polarizationindependent metamaterial absorber for solar energy harvesting applications. Physica E: Lowdimensional Systems and Nanostructures, 90: 1-6 
Bakır M, Karaaslan M, Dincer F, Delihacioglu K, Sabah C, 2015. Perfect metamaterial absorber-based energy harvesting and sensor applications in the industrial, scientific, and medical band. Optical Engineering, 54(9): 097102.

Bakır M, Karaaslan M, Dincer F, Delihacioglu K, Sabah C, 2016. Tunable perfect metamaterial absorber and sensor applications. Journal of Materials Science: Materials in Electronics, 27: 12091-12099.

Bakır M, 2018. Metamaterial based multiband energy harvesting application. Journal of Balıkesir University Institute of Science and Technology, 20(1): 517-538.

Bai Q, Langley R, 2009. Wearable EBG antenna bending and crumpling. 2009 Loughborough Antennas and Propagation Conference, 16-17 Nevember 2009, Loughborough University. UK

Gupta B, Sankaralingam S, Dhar S, 2010. Development of wearable and implantable antennas in the last decade: A review, 2010 10th Mediterranean Microwave Symposium, 25-27 August 2010. Northern Cyprus.

Kim JY, Ha SJ, Kim D, Lee B, Jung CW, 2012. Reconfigurable beam steering antenna using U-slot fabric patch for wrist-wearable applications. Journal of Electromagnetic Waves and Applications, 26(11): 1545-1553.

Liu D, Pfeiffer J, Grzyb J, Gaucher B, 2009. Advanced Milimeter-Wave Tecnologies: Antennas, Packaging and Circuts, chapter 5, Wiley. 163-232 p.

Mantash M, Tarot AC, Collardey S, Mahdjoubi K, 2012. Investigation of flexible textile antennas and AMC reflectors. International Journal of Antennas and Propagation, 2012: 1-10.

Moroz A, 2009. Some negative refractive index material headlines long before Veselago work and going back as far as to 1905, http://www.wave-scattering.com/negative.html, 07 February 2020.

Ozbay E, Aydin K, 2008. Negative refraction and imaging beyond the diffraction limit by a twodimensional left-handed metamaterial. Photonics and Nanostructures-Fundamentals and Applications, 6(1): 108-115

Pendry JB, 2000. Negative refraction makes a perfect lens. Physical review letters, 85(18): 3966.

Pendry JB, Holden AJ, Robbins DJ, Stewart WJ, 1999. Magnetism from conductors and enhanced nonlinear phenomena. IEEE transactions on microwave theory and techniques, 47(11): 2075-2084

Sabah C, Dincer F, Karaaslan M, Bakir M, Unal E, Akgol O, 2015. Biosensor applications of chiral metamaterials for marrowbone temperature sensing. Journal of Electromagnetic Waves and Applications, 29(17): 2393-2403.

Salahun E, Queffelec P, Floch ML, Gelin P, 2001. A broadband permeameter for 'in situ' meassurument of rectangular samples. IEEE Transactions on Magnetics, 37(4): 2743-2745.

Salonen P, Rahmat-Samii Y, Schaffrath M, Kivikoski M, 2004. Effect of textile materials on wearable antenna performance: a case study of GPS antennas, IEEE Antennas and Propagation Society Symposium, 20-25 June 2004, Monterey. California.

Sankaralingam S, Gupta B, 2010. Use of Electro-Textiles for Development of Wibro Antennas. Progress In Electromagnetics Research, 16: 183-193.

Shelby RA, Smith DR, Schultz S, 2001. Experimental verification of a negative index of refraction. 292(5514): 77-79.

Soh PJ, Vandenbosch GAE, Ooi SL, Rais, NHM, 2012. Design of a broadband all-textile slotted PIFA. IEEE Transactions on Antennas and Propagation, 60(1): 379-384.

Tetik E, Tetik G, 2018. The effect of a metamaterial based wearable monopole antenna on the human body. Celal Bayar Üniversitesi Fen Bilimleri Dergisi, 14(1): 93-97.

Tetik, E., Erdiven, U., 2018. Functional Pressure and Density Sensor Design Based on Metamaterial Absorber. Çukurova Univ. J. Fac. Eng. Archit. 33, 23-30. 
Tronquo A, Rogier H, Hertleer C, Van Langenhove, L, 2006. Robust planar textile antenna for wireless body LANs operating in $2.45 \mathrm{GHz}$ ISM band. Electronics letters, 42(3): 142-143.

Tronquo A, Rogier H, Hertleer C, Van Langenhove L, 2006. Applying textile materials for the design of antennas for wireless body area networks, 2006 First European Conference on Antennas and Propagation, 6-10 November 2006, Nice. France.

Xiao Z, Lv F, Li W, Zou H, Li C, 2020. A three-dimensional ultra-broadband and polarization insensitive metamaterial absorber and application for electromagnetic energy harvesting. Waves in Random and Complex Media, 1-9.

Veselago VG, 2002. Electrodynamics of Media with Simultaneously Negative Electric Permittivity and Magnetic Permeability. Advances in Electromagnetics of Complex Media and Metamaterials, Springer, Dordrecht, 83p.

Yan S, Guy AE, 2016. Radiation pattern-reconfigurable wearable antenna based on metamaterial structure. IEEE Antennas and wireless propagation Letters, 15: 1715-1718.

Zhu S, Langley R, 2009. Dual-band wearable textile antenna on an EBG substrate. IEEE transactions on Antennas and Propagation, 57(4): 926-935. 